\title{
G protein-coupled receptors in stem cell maintenance and somatic reprogramming to pluripotent or cancer stem cells
}

\author{
Hye Yeon Choi, Subbroto Kumar Saha, Kyeongseok Kim, Sangsu Kim, Gwang-Mo Yang, BongWoo Kim, Jin-hoi Kim E \\ Ssang-Goo Cho* \\ Department of Animal Biotechnology, Animal Resources Research Center, and Incurable Disease Animal Model and Stem Cell Institute \\ (IDASI), Konkuk University, Seoul 143-701, Korea
}

\begin{abstract}
G protein-coupled receptors (GPCRs) are a large class of transmembrane receptors categorized into five distinct families: rhodopsin, secretin, adhesion, glutamate, and frizzled. They bind and regulate $80 \%$ of all hormones and account for $20-50 \%$ of the pharmaceuticals currently on the market. Hundreds of GPCRs integrate and coordinate the functions of individual cells, mediating signaling between various organs. GPCRs are crucial players in tumor progression, adipogenesis, and inflammation. Several studies have also confirmed their central roles in embryonic development and stem cell maintenance. Recently, GPCRs have emerged as key players in the regulation of cell survival, proliferation, migration, and self-renewal in pluripotent (PSCs) and cancer stem cells (CSCs). Our study and other reports have revealed that the expression of many GPCRs is modulated during the generation of induced PSCs (iPSCs) or CSCs as well as during CSC sphere formation. These GPCRs may have crucial roles in the regulation of selfrenewal and other biological properties of iPSCs and CSCs. This review addresses the current understanding of the role of GPCRs in stem cell maintenance and somatic reprogramming to PSCs or CSCs. [BMB Reports 2015; 48(2): 68-80]
\end{abstract}

\section{INTRODUCTION}

Many tissues of the body-for example, skin, liver, and epithelium - not only repair themselves but also self-renew, a property found mainly in stem cells (1). Embryonic stem cells (ESCs) have an even greater potential for self-renewal and differentiation. Recently, mouse and human fibroblasts were successfully reprogrammed into pluripotent stem cells (PSCs)

${ }^{*}$ Corresponding author. Tel: +82-2-450-4207; Fax: +82-2-4501044; E-mail: ssangoo@konkuk.ac.kr

http://dx.doi.org/10.5483/BMBRep.2015.48.2.250

Received 18 November 2014

Keywords: Cancer stem cells (CSC), G protein-coupled receptor (GPCR), Induced pluripotent stem cell (iPSC), Somatic reprogramming, Stem cell maintenance with the introduction of a diverse set of stem cell-related transcription factors including Oct4, Sox2, KIf4, and c-Myc $(2,3)$. These induced PSCs (iPSCs) derived from somatic fibroblasts had genetic, epigenetic, and developmental features that were highly similar to those of ESCs. Although ESCs and iPSCs are considered unlimited cell sources for regenerative medicine, techniques for maintaining undifferentiated ESC or iPSCs remain inefficient, which can lead to inhomogeneous cell populations.

Tumor cells are assumed to include a population of cells responsible for initiating tumor development and growth, with the capacity to metastasize and reoccur (4). Because of their similarities to stem cells, these cells have been named cancer stem cells (CSCs). CSCs have properties such as self-renewal, heterogeneity, and resistance to apoptosis. CSCs likely arise from stem cells, and the transformation of normal stem cells into CSCs may be due to the accumulation of genetic modifications such as mutations in oncogenes, suppressor genes, and mismatch repair genes or a result of epigenetic alterations such as abnormal methylation and histone modifications (5).

The cell survival, proliferation, migration, and self-renewal of PSCs and CSCs are regulated by various signaling molecules including G protein-coupled receptors (GPCRs) (6). GPCRs, also known as seven-transmembrane domain receptors, 7TM receptors, heptahelical receptors, serpentine receptors, and G protein-linked receptors (GPLR), are a large class of transmembrane (TM) receptors that conduct extracellular signals into cells by coupling with guanine nucleotide-binding proteins (G proteins) and interacting with a diverse set of ligands. They are by far the largest family of cell surface molecules, and they modulate key physiological functions, including neurotransmission, hormone and enzyme release, immune response, and blood pressure regulation. Their signaling converges on common downstream effectors and modulators, such as G proteins, arrestins, and GPCR kinases/G protein-coupled receptor kinases. Most GPCRs activate one or multiple G $\alpha$ proteins, which can be subdivided into four major families: Goi, Go12, Gos, and Goq (7). GPCRs act more as molecular regulators than on-off switches, so the engagement of different $G$ proteins and the duration of signaling may differ not only among GPCRs but also for a given GPCR depending on the ligand and 
cellular environment (8). Considerable evidence now exists demonstrating the important roles of various GPCRs in regulating the biological properties of PCSs or CSCs.

Recently, we analyzed the expression profiles of GPCRs during somatic reprogramming to iPSCs or CSCs and during CSC sphere formation (Fig. 1 and Table 1). More than 106 GPCRs were over-expressed in the PCSs or CSCs, whereas the expression of 22 GPCRs was down-regulated during somatic reprogramming to iPSCs. Eighty-one GPCRs were differentially expressed during somatic reprogramming to iPSCs, and the expression of 195 GPCRs was either up- or down-regulated during somatic reprogramming to CSCs and sphere formation of CSCs. These data suggest that various GPCRs may have key roles in somatic reprogramming to iPSCs or CSCs and may be involved in the regulation of self-renewal and other biological properties of PCSs or CSCs. Recently, much evidence has ac- cumulated supporting the specific roles of GPCRs in somatic reprogramming or transformation to iPSCs or CSCs. In the following section, we review the general role of GPCR signaling pathways and the current understanding of the role of GPCRs in stem cell maintenance and somatic reprogramming to PCSs or CSCs.

\section{GENERAL ROLE OF GPCR SIGNALING PATHWAYS}

GPCRs bind and regulate the effects of $80 \%$ of all hormones in the body and account for $20-50 \%$ of the pharmaceuticals on the current market (9). Members of the GPCR superfamily have seven transmembrane (TM) domains as well as extracellular $\mathrm{N}$ and cytosolic C-termini (10). The exact size of the GPCR superfamily is unknown, but nearly 800 GPCR genes comprise $3-5 \%$ of the human genome. The superfamily has traditionally
A
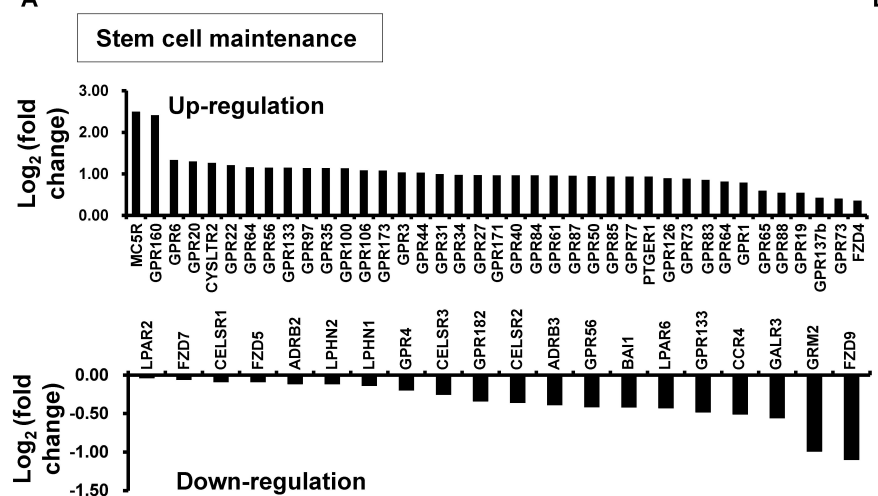

C

CSC Sphere formation

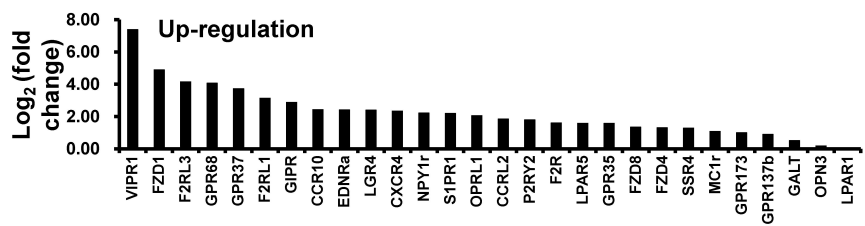

\section{Somatic Reprogramming to iPSCs}
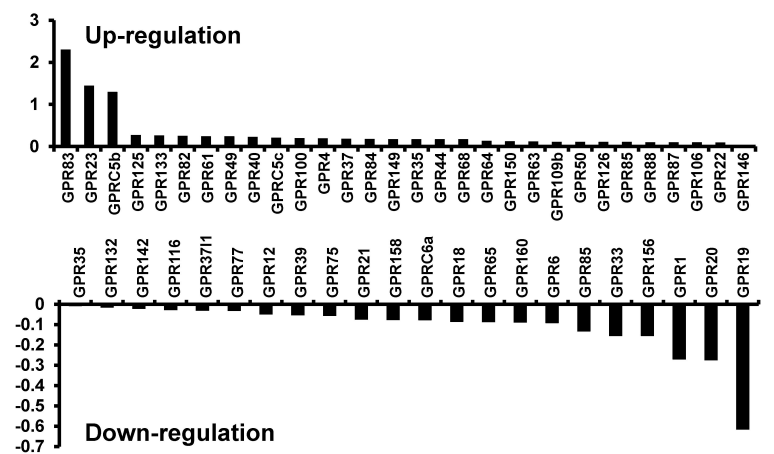

D

\section{Somatic Reprogramming to CSCs}

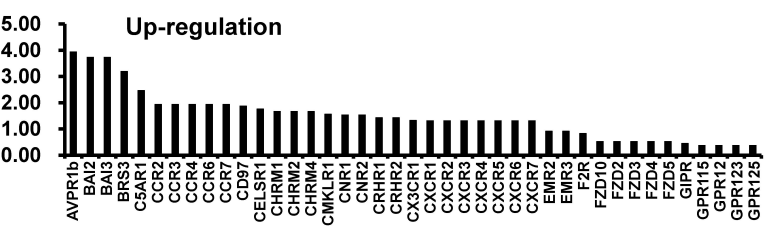

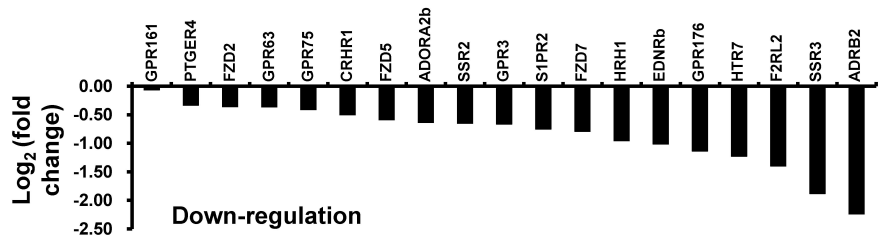

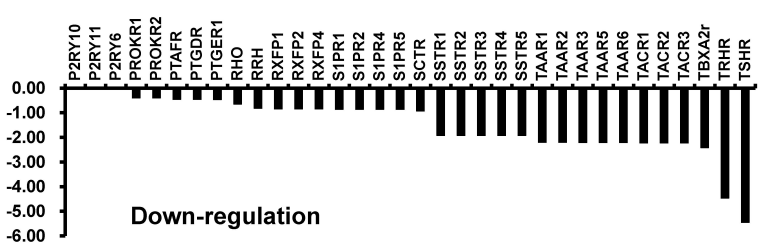

Fig. 1. Changes in G protein-coupled receptor (GPCR) expression in stem cell maintenance and/or during somatic reprogramming to iPSCs or CSCs. The transcriptional profile of the selected GPCR family was analyzed using high-throughput RNA sequencing. (A) GPCRs showing up- or down-regulated expression during stem cell maintenance. (B) GPCRs showing up- or down-regulated expression during somatic reprogramming to iPSCs. (C) GPCRs showing up- or down-regulated expression during CSC sphere formation. (D) GPCRs showing up- or down-regulated expression during malignant transformation (somatic reprogramming) to CSCs. 
G protein-coupled receptors in stem cell maintenance and somatic reprogramming to pluripotent or cancer stem cells Hye Yeon Choi, et al.

Table 1. Differential GPCRs showing up- or down-regulated expression during stem cell maintenanceor somatic reprogramming to iPSCs or CSCs

\begin{tabular}{llcc}
\hline Condition & Classification & Family & Subfamily (Gene) \\
\hline Stem cell & CLASS A & Rhodopsin-like & A1 (GPR137b, CCR4), A2 (CXCR7, GPR182), A4 (GPR1, GPR22), A5 (GPR100,
\end{tabular}

Stem cell CLASS A Rhodopsin-like A1 (GPR137b, CCR4), A2 (CXCR7, GPR182), A4 (GPR1, GPR22), A5 (GPR100,

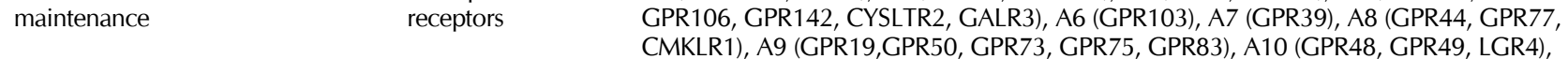
CMKLR1), A9 (GPR19,GPR50, GPR73, GPR75, GPR83), A10 (GPR48, GPR49, LGR4),
A11 (GPR40, GPR43, GPR81, GPR82, GPR109b), A12 (GPR34, GPR87, GPR171), A13 (GPR3, GPR6, GPR12, MC5R, S1PR2, LPAR1, LPAR2), A14 (PTGER1), A15 (LPAR4, F2RL1, LPAR5, F2R, GPR4, LPAR6, GPR18, GPR20, GPR35, GPR65, GPR68, GPR119, GPR132), A17 (ADRB1, ADRB2, ADRB3), A18 (GPR21, GPR27, GPR45, GPR61, GPR63, GPR84, GPR85, GPR88, GPR173, ADORA2b), Orphan (GPR23, GPR26, GPR33, GPR35, GPR37, GPR54, GPR83, GPR135, GPR146, GPR149, GPR150, GPR151, GPR152, GPR153, GPR160)

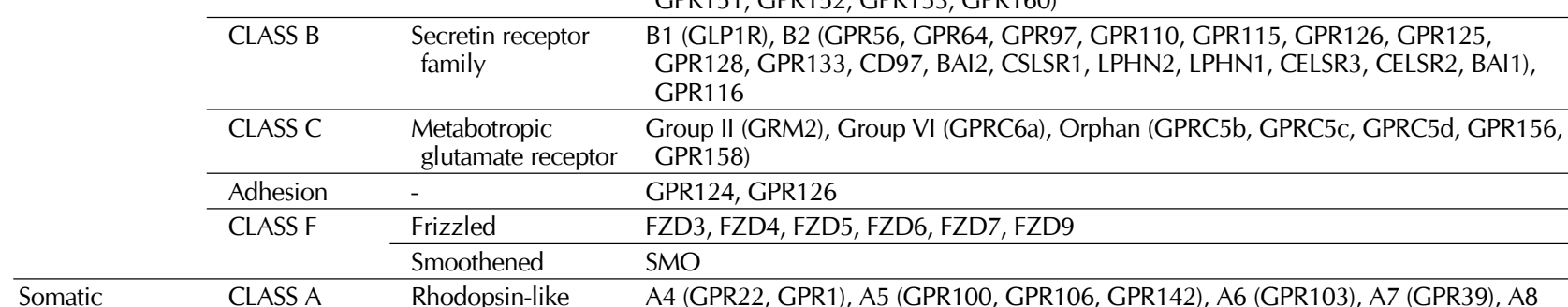

$\begin{array}{lll}\text { reprogramming } & \begin{array}{c}\text { Rhodopsin-like } \\ \text { receptors }\end{array} & \text { (GPR44, GPR77), A9 (GPR50, GPR73, GPR75, GPR19), A10 (GPR49, GPR48), A11 }\end{array}$ to iPSCs $\quad$ (GPR82, GPR40, GPR43, GRP109b, GPR81), A12 (GPR87, GPR34, GPR171), A13 (GPR3, GPR12, GPR6), A15 (GPR4, GPR68, GPR119, GPR35, GPR132, GPR18, GPR65, GPR20), A18 (GPR61, GPR84, GPR85, GPR45, GPR63, GPR88, GPR173, GPR27, GPR21), Orphan (GPR141, GPR23, GPR26, GPR33, GPR35, GPR37, GPR54, GPR135, GPR149, GPR150, GPR151, GPR152, GPR153, GPR160)

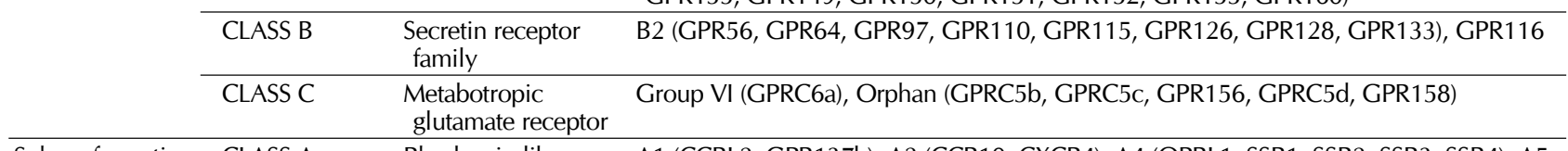

\begin{tabular}{lll}
\hline Sphere formation & CLASS A & Rhodopsin-like
\end{tabular} A1 (CCRL2, GPR137b), A2 (CCR10, CXCR4), A4 (OPRL1, SSR1, SSR2, SSR3, SSR4), A5 $\begin{array}{ll}\text { of CSCs } & \text { receptors } \\ \text { (GALT, LTB4R), A6 (GPR176), A7 (EDNRA, EDNRB, GPR37, GPR39), A10 (LGR4), }\end{array}$ A11 (P2RY2), A13 (LPAR1, LPAR2, S1PR1, S1PR2, MC1R, GPR3), A14 (PTGER4), A15 (LPAR5, F2RL1, F2RL2, F2RL3, GPR68, GPR35, F2R), A16 (OPN3), A17 (ADRB2), A18 (HRH1, ADORA1, ADORA2B, GPR64, GPR161, GPR173), A19 (HTR7)

\begin{tabular}{llll}
\cline { 2 - 3 } & CLASS B & $\begin{array}{c}\text { Secretin receptor } \\
\text { family }\end{array}$ & B1 (CRHR1, GIPR, VIPR1) \\
\cline { 2 - 4 } & CLASS F & Frizzled & FZD1, FZD2, FZD4, FZD5, FZD6, FZD7, FZD8 \\
\hline Somatic & CLASS A & Rhodopsin-like & A1 (CCR2, CCR3, CCR4, CR8, CCRL2, CX3CR1, GPR137b), A2 (CCR6, CCR7, CCR9,
\end{tabular}

Somatic CLASS A Rhodopsin-like A1 (CCR2, CCR3, CCR4, CR8, CCRL2, CX3CR1, GPR137b), A2 (CCR6, CCR7, CCR9,

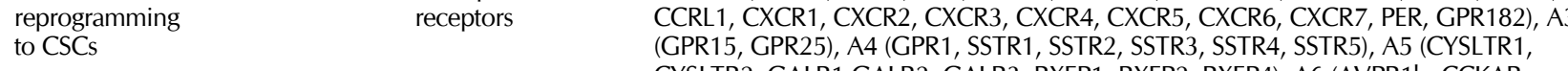
CYSLTR2, GALR1 GALR2, GALR3, RXFP1, RXFP2, RXFP4), A6 (AVPR1b, CCKAR, CCKBR, GNRHR, GPR176, GPR22, HCRTR2, QRFPR), A7 (BRS3, EDNRB, GHSR, GPR37, GPR39, GPRP, TRHR), A8 (C3AR1, CMKLR1, FPR1, GPR1, GPR32, GPR77), A9 (GPR19, GPR50, PPYR1, PRLHR, PROKR1, PROKR2, TACR1, TACR2, TACR3), A10 (TSHR), A11 (FFAR1, FFAR2, FFAR3, GPR31, GPR82, HCAR1, HCAR2, HCAR3, OXGR1, P2RY1, P2RY11, P2RY2, P2RY4, P2RY6, PWRY8), A12 (GPR171, GPR87, P2RY13, P2RY14, PTAFR), A13 (CNR1, CNR2, GPR12, GPR3, GPR6, S1PR1, S1PR2, S1PR4, S1PR5), A14 (PTGDR, PTGER1, PTGER2, PTGER3, PTGER4, PTGER, PTGIR, TBXA2R), A15 (F2R, F2RL1, F2RL2, F2RL3, GPR132, GPR17, GPR183, GPR35, GPR65, P2RY10), A16 (RGR, RHO, RRH), A17 (DRD1, DRD2, DRD4, HRH2, HTR2A, HTR2B, HTR2C, HTR6, TAAR1, TAAR2, TAAR3, TAAR5, TAAR6), A18 (CHRM1, CHRM2, CHRM4, GPR161, GPR173, GPR21, GPR27, GPR45, GPR52, GPR61, GPR63, GPR78, GPR85, GPR88, HRH1, HRH3, HRH4), A19 (HTR1D, HTR1F, HTR4, HTR7)

\begin{tabular}{|c|c|c|}
\hline CLASS B & $\begin{array}{l}\text { Secretin receptor } \\
\text { family }\end{array}$ & $\begin{array}{l}\text { B1 (CRHR1, CRHR2, GCGR, GHRHR, GIPR, GLP1R, GLP2R, SCTR), B2 (BAI2, BAI3, } \\
\text { CD97, CELSR1, ELTD1, EMR1, EMR2, EMR3, GPR110, GPR111, GPR113, GPR114, } \\
\text { GPR115, GPR123, GPR125, GPR126, GPR128, GPR144, GPR56, GPR64) }\end{array}$ \\
\hline CLASS C & $\begin{array}{l}\text { Metabotropic } \\
\text { glutamate receptor }\end{array}$ & Group I (GRM1, GRM5), Group II (GRM4, GRM7, GRM8), Group III (GRM2, GRM3) \\
\hline CLASS F & Frizzled & FZD2, FZD3, FZD4, FZD5, FZD6, FZD7, FZD8, FZD9, FZD10 \\
\hline
\end{tabular}



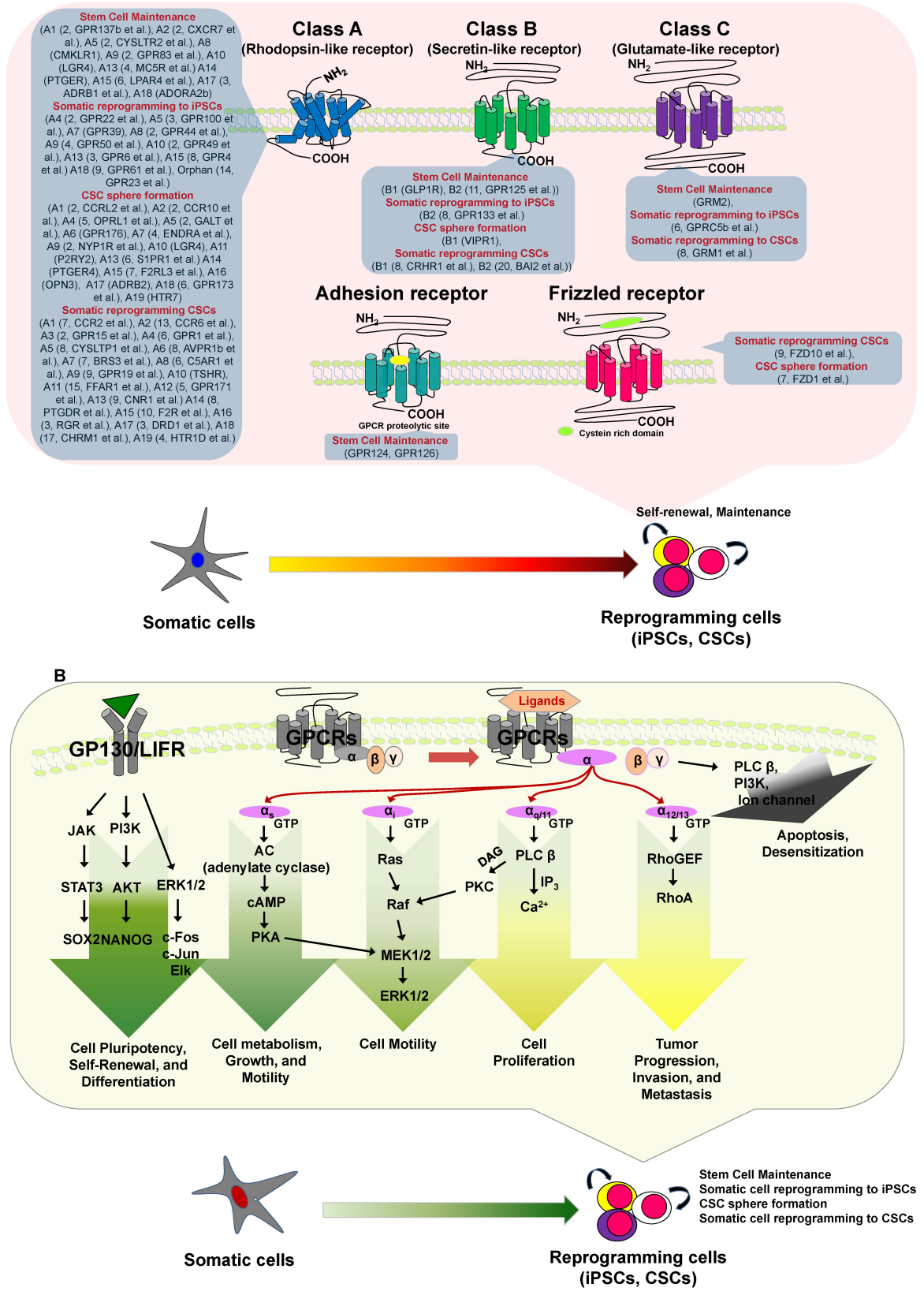

Fig. 2. Differential GPCRs and GPCR signaling, which may be involved in stem cell maintenance and/or during somatic reprogramming to iPSCs or CSCs. (A) The GPCR superfamily has traditionally been divided into three major families: class A/rhodopsin-like receptors, class B/secretin-like receptors, and class C/glutamate-like receptors. Recent bioinformatics analyses have updated the phylogenetic characterization to five distinct families: glutamate, rhodopsin, adhesion, frizzled and secretin (GRAFS classification system). (B) Various ligands bind GPCRs to stimulate various $G$ proteins. GPCRs interact with heterotrimeric $G$ proteins composed of $\alpha, \beta$, and $\gamma$ subunits that are guanosine diphosphate bound in the resting state. Most GPCRs activate one or multiple G $\alpha$ proteins, which can be subdivided into four major families:

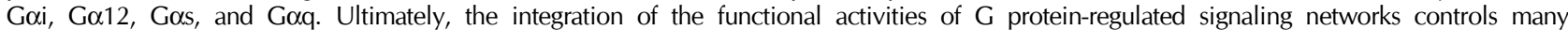
cellular functions, and the aberrant activities of $G$ proteins and their downstream target molecules can contribute to various cellular mechanisms, including roles in stem cell maintenance and somatic reprogramming to iPSCs or CSCs. (B) Activation of pluripotency and differentiation pathways by GPCRs. 
been divided into three major families - class A/rhodopsin-like receptors, class $\mathrm{B} /$ secretin-like receptors, and class C/glutamate-like receptors. The largest class is class A, which accounts for almost $85 \%$ of the GPCR genes. Recent bioinformatics analyses have updated the phylogenetic characterization to five distinct families: glutamate, rhodopsin, adhesion, frizzled (FZD), and secretin (i.e., the GRAFS classification system) (Fig. 2) (11, 12).

GPCR stimulation triggers the activation of heterotrimeric G proteins as guanosine triphosphate (GTP) replaces guanosine diphosphate on the $\mathrm{G} \alpha$ subunit, promoting its dissociation from the G $\beta \gamma$ subunits. Both $\alpha$-GTP-bound and G $\beta \gamma$ subunit complexes then stimulate multiple downstream signaling cascades (7), including the rapid generation of multiple second messengers. For example, Gos stimulates adenylyl cyclase, increasing the cytosolic levels of cyclic adenosine monophosphate (CAMP), whereas Goi inhibits adenylyl cyclase and subsequently decreases cAMP levels (13). Members of the Goq family activate phospholipase- $C \beta$, which cleaves phosphatidylinositol-4, 5-bisphosphate into diacylglycerol and inositol 1,4,5-trisphosphate; the latter increases cytosolic calcium (14). The targets of these diffusible second messengers include ion channels, calcium-sensitive enzymes, and kinases such as cAMP-dependent kinase, protein kinase $\mathrm{C}$, cyclic guanosine monophosphate-dependent kinase, and calcium-calmodulin regulated kinases. Many of these kinases contribute to cancer progression and metastasis (15). Although GPCRs can stimulate multiple diffusible second messenger-generating systems, their capacity to promote normal and aberrant cell proliferation often relies on the persistent activation of phosphatidylinositol 3'-kinase/protein kinase B, Ras and Rho GTPases, and mitogen-activated protein kinase cascades, thereby regulating the activity of nuclear transcription factors and co-activators such as JUN and FOS (16). The final biological outcome of GPCR activation is a result of the integration of the GPCR-initiated biochemical response networks in each cellular and environmental context (8).

\section{GPCRs IN STEM CELL MAINTENANCE}

PCSs have great potential to aid in the understanding of the early development and treatment of human diseases and tissue disturbances. We found that optimizing the culture conditions can enhance the pluripotency of stem cells $(17,18)$. The expression of certain membrane proteins, including GPCRs, result in the regulation of cell morphology, polarity and the migration of stem cells (19). Extensive evidence suggests that GPCRs show dramatically different expressions when cells differentiate, but the roles of GPCRs in stem cells maintenance are poorly understood (20). The self-renewal and pluripotency of PCSs are regulated by several Gs- and Gi-coupled GPCR signaling pathways (21). Signaling mediated by the G proteins of the Gi subfamily affects the morphology of iPSCs (20). Gs signaling also promotes both proliferation and pluripotency of
ESCs (21). Especially, during in vitro neural differentiation, distinct GPCR genes are specifically expressed at each differentiation stage (22). The specific roles of GPCRs from the five families (glutamate, rhodopsin, adhesion, FZD, and secretin) are reviewed in the following section.

The glutamate family includes metabotropic glutamate receptors (mGluRs), $\gamma$-aminobutyric acid B (GABAB) receptors, taste receptors, and related orphan receptors (12). Glutamate is abundant in the human body and is particularly prominent in the nervous system. It is present in over $50 \%$ of nervous tissue and is the most abundant excitatory neurotransmitter in the central nervous system (CNS), playing a key role in the synaptic plasticity required for learning and memory. It is also the precursor for GABA, the primary inhibitory neurotransmitter in the mammalian CNS. Glutamate receptors can be divided into two groups according to the mechanism by which their activation gives rise to a postsynaptic current. Ionotropic glutamate receptors (iGluRs) form the pores for their own ion channels while metabotropic glutamate receptors (mGluRs) indirectly activate ion channels on the plasma membrane through a signaling cascade that involves $G$ proteins. mGluRs are responsible for the glutamate-mediated postsynaptic excitation of neural cells and are also reported to play an important role in the cell growth and differentiation of ESCs. The mGluR family is composed of eight subtypes, classified as mGluR1 (GRM1) to mGluR8 (GRM8), which are further divided into subtypes, such as mGluR7a and mGluR7b. Receptor types are grouped based on receptor structure and physiological activity. The mGluRs in group I, comprised of mGluR1 and mGluR5, are stimulated most strongly by the excitatory amino acid analog L-quisqualic acid and couple to Gq proteins (23). Stimulation of the receptors causes activation of phospholipase $\mathrm{C}$, leading to the formation of inositol 1,4,5-triphosphate and diacylglycerol. These receptors are also associated with $\mathrm{Na}^{+}$and $\mathrm{K}^{+}$ channels. The receptors in group II, mGluRs 2 and 3, and group III, which consist of mGluRs 4, 6, 7, and 8, prevent the formation of CAMP by activating a $G$ protein that inhibits the enzyme adenylyl cyclase. Receptors in groups II and III, mGluR 2, 3, 4, 6, and 7, interact with Gi G proteins to reduce the activity of postsynaptic potentials, both excitatory and inhibitory, in the cortex. The activation of mGluR 5 promotes self-renewal by inhibiting glycogen synthase kinase-3 $\beta$ (GSK-3ß) and leukemia inhibitory factor (LIF) activation of phosphatidylinositol 3'-kinase (24). During ESC differentiation, mGluR5 expression decreases and mGluR4 expression is upregulated (25). The differentiation of neural stem cells is also suppressed upon activation of mGlu3R via mitogen-activated protein kinase-dependent inhibition of bone morphogenetic protein signaling (26).

GABA plays the principal role in neuronal excitability reduction throughout the nervous system. Although GABAA receptors are ligand-gated ion channels, the metabotropic GABAB receptors are GPCRs (glutamate GPCR family) which interact with GABA and have obvious regulatory roles in neu- 
ronal activity. ESCs synthesize GABA and express functional GABAA receptors and GABAB receptors (27). Both GABAR types synergistically trigger intracellular calcium increase and the interplay between the GABARs and downstream effectors differentially modulates ESC proliferation and differentiation through selective activation of second messenger signaling cascades. Moreover, ESCs are reported to possess GABAergic circuit machinery and establish a GABA niche via release of the transmitter (28).

The rhodopsin family is the largest GPCR family, comprising nearly $85 \%$ of GPCRs (12). Chemokine receptors, part of the rhodopsin GPCR family, comprise a large subfamily of seven TM proteins that bind one or more chemokines, which are small cytokines that typically have chemotactic activity for leukocytes (29). The chemokine receptor C-X-C chemokine receptor type 4 (CXCR4), also known as fusin or CD184 (cluster of differentiation 184), may have an important role in stem cell maintenance. CXCR4 is an alpha-chemokine receptor specific for stromal cell-derived factor-1 $\alpha$ (SDF-1), which is also called CXCL12 and important in hematopoietic stem cell homing to the bone marrow and in hematopoietic stem cell quiescence (30). It regulates the induction of proliferation, trafficking, locomotion, and adhesion of PCSs and has a role in the homing of engrafted stem cells (31). CXCR4 also regulates the migration of stem cells and has an important role in stem cell-based therapies for multiple sclerosis. Currently, there are two known receptors for SDF-1: CXCR4 and CXCR7. Recent reports indicate that both CXCR4 and the SDF-1 scavenging receptor, CXCR7/ACKR3 (CXCR7), are expressed in stem cells and play important roles in stem cell therapy for remyelination (32).

The cannabinoid receptors are also members of the rhodopsin family of GPCRs and are activated by endogenous ligands that include anandamide, anandamide derivatives (2-arachidonoyl glycerol and noladin ether), virodhamine, and $\mathrm{N}$-arachidonoyldopamine (33). There are currently two known subtypes of cannabinoid receptors, termed CB1 and CB2. CB1 receptor expression is abundant in brain CNS and is involved in a variety of physiological processes including memory, appetite, mood, and pain-sensation. CB2 receptors are mostly expressed in hematopoietic cells, in T and B cells of the immune system, and in macrophages. Additional cannabinoid receptors that are non- $\mathrm{CB}_{1}$ or non- $\mathrm{CB}_{2}$ are expressed in endothelial cells and in the CNS. Several orphan GPCRs such as GPR18, GPR55, and GPR119 have been suggested to serve as cannabinoid receptors (34). Significant induction of CB1 and CB2 receptors in addition to the expression of endocannabinoids, the ligands for both CB1 and CB2, are observed during the hematopoietic differentiation of ESCs. Treatment of ESCs with the exogenous cannabinoid ligand led to the increased hematopoietic differentiation of ESCs, while addition of the CB antagonists resulted in suppression of the embryoid body formation, suggesting that endocannabinoids and $\mathrm{CB}$ receptors are involved in the maintenance of ESCs. In addition, cannabinoid agonists induced the chemotaxis of embryoid bodies, which was specifically inhibited by CB1 and CB2 antagonists (35). Recently, in addition to their roles in survival, homing, and migration of stem cells, the potential role of CB2 receptors as mediators of the anti-inflammatory properties of human mesenchymal stromal cells was reported (36). Cannabinoid signaling regulates cell proliferation, differentiation and survival and CB receptors are expressed and functional during developmental stages (37). After the receptor is activated by cannabinoids, generated naturally inside the body (endocannabinoids) or introduced into the body as cannabis, CB receptors interact with Gi G proteins and primarily inhibit adenylyl cyclase, leading to the suppression of cyclic AMP production. $\mathrm{CB}$ receptors-mediated regulation of various signaling pathways including protein kinase A and C, Raf1, and MAPK, may be involved in the maintenance of ESCs (35). CB receptors also have roles in regulating the fate of stem cells in ectoderm-derived neural progenitors and mesoderm-derived hematopoietic progenitors. They regulate ESC survival and differentiation, and thus may affect the formation of the three germ layers (ectoderm, mesoderm and endoderm). CB1 and CB2 receptors are present in neural progenitors and are reported to control their self-renewal, proliferation and differentiation in opposite patterns. CB1 receptor has a prosurvival function in mesenchymal stem cells and also plays a role in osteogenesis (38).

Lysophosphatidic acid (LPA) activates six rhodopsin family GPCRs, namely LPA1 (EDG2), LPA2 (EDG4), LPA3 (EDG7), LAP4 (p2y9/GPR23), LPA5 (GPR92), and LPA6 (GPR87). It has also been identified as an agonist for other orphan GPCRs (PSP24, GPR87, and GPR35) as well as at receptors of the nuclear hormone peroxisome proliferator-activated receptor $\gamma$ (39). LPA is reported to be a positive regulator of the self-renewal and pluripotency of PCSs (40). LPA may regulate ESC maintenance, self-renewal and somatic cell reprogramming to iPSCs via ERK-mediated activation of c-fos or by overexpression of pluripotent transcription factors, such as c-Myc (41). The mode of action in human ESCs (hESCs) also involves Gi- and ERK-dependent mechanisms (42). Expression of specific LPA receptors has been reported in stem cells, such as the expression of LPA1 and LPA2 in hematopoietic cells (43) and the expression of LPA1-3 in mesenchymal stromal cells (44).

Sphingosine 1-phosphate (S1P), a signaling sphingolipid, activates five rhodopsin family GPCRs known as S1P receptors (S1PR1-S1PR5) (45). The S1P receptors regulate fundamental biological processes such as cell proliferation, angiogenesis, migration, cytoskeleton organization, endothelial cell chemotaxis, immune cell trafficking and mitogenesis. Like LPA, S1P also positively regulates the self-renewal and pluripotency of PCSs via ERK activation (40). Expression studies in ESCs have revealed that LPA1-3 and 5 together with S1P1-5 are expressed in ECSs and that LPA and S1P increase the expression of pluripotency genes and stimulate cell proliferation (46) through a Gi-ERK-dependent pathway (40).

Rhodopsin family somatostatin receptors (SSTR1-5) are also involved in the regulation of stem cell maintenance (47). So- 
matostatin inhibits the release of many hormones and other secretory proteins at various sites by interacting with SSTRs that are expressed in a tissue-specific manner. SSTR2, which is expressed at the highest levels in the cerebrum and kidneys, interacts with SHANK2 (SH3 and multiple ankyrin repeat domains protein 2), a synaptic protein involved in the maintenance of the typical morphology of ESCs. Addition of the SSTR2 agonist octreotide or seglitide leads to promotion of the self-renewal of ESCs, whereas the SSTR2 antagonist S4 results in dose-dependent suppression of the SSTR2 agonist-induced self-renewal. Furthermore, knock-down of SSTR2 significantly decreases the self-renewal of ESCs and reduces the phosphorylation and nuclear localization of STAT3, suggesting that SSTR2 contributes to the self-renewal of ESCs via activation of the STAT3 pathway.

Next, adhesion GPCRs are a class of 33 human protein receptors that can be divided into 8 groups, with two additional adhesion GPCRs, VLGR1 and GPR128 (48). They are broadly distributed in leukocytes, neurons, embryonic and larval cells, cells of the reproductive tract, and a variety of tumors. Adhesion GPCRs were also reported to be involved in embryogenesis and development (49). Adhesion family GPCRs have adhesion motifs containing long $\mathrm{N}$-termini that are often involved in protein-protein interactions and that mediate signaling. Many adhesion GPCRs undergo proteolytic events posttranslationally at highly conserved Cys-rich motifs known as GPCR proteolysis sites (GPS), which are located next to the first transmembrane region. Once this protein is cleaved, the pieces are expressed at the cell surface as a heterodimer. A Group VIII adhesion GPCR, GPR56, has been shown to be cleaved at the GPS site. The receptor became constitutively active after cleavage and an upregulation of G $\alpha 12 / 13$ was observed. GPR56 has a known ligand, Collagen III, which is involved in neural migration. The interaction between GPR56 and Go12/13 results in the induction of Rho-mediated cytoskeletal changes and plays a role in stem cell maintenance and differentiation (50). Additionally, the cadherin/CELSR subgroup of adhesion receptors, Celsr1-3, have key roles in migration and proliferation during development (51). GPR125, which is a Group III adhesion receptor, may also be involved in stem cell maintenance (52).

The Wnt signaling pathways are a group of signaling pathways composed of proteins that pass Wnt-binding signals from the outside to the inside of a cell through FZD cell surface receptors $(53,54)$. The FZD family receptors show the typical structural characteristics of GPCRs and are activated by the Wnt family, which comprises a diverse family of secreted cysteine-rich lipid-modified signaling glycoproteins with fundamental functions in ontogeny and tissue homeostasis. Previously, the FZD family receptors were not recognized as GPCRs. However, they were officially classified as a GPCR family in 2005 by the International Union of Pharmacology $(53,54)$. The Wnt and FZD proteins are highly conserved across species and 11 FZD and 19 Wnt genes have been identified (55).
Three Wnt/FZD signaling pathways have been characterized; the canonical Wnt pathway, the noncanonical planar cell polarity pathway, and the noncanonical Wnt/calcium pathway are implicated in regulation embryonic development and stem cell maintenance. The Wnt signaling pathway contributes to the maintenance of pluripotency in ESCs (56). ESCs express members of the FZD7 receptor family and secrete FZD-related proteins encoding soluble Wnt antagonists (57). Moreover, activation of the Wnt pathway by wnt3a or the GSK-3 inhibitor 6-bromoindirubin-3'-oxime leads to self-renewal and pluripotency (58). Expression levels of Wnt3a and FZD7 in ESCs is higher than those in differentiated cell types. Knockdown of FZD7 also leads to obvious changes in the typical morphology of ESCs and decreases in Oct4 expression, implying that the Wnt3a/FZD7 pathway is important for ESC maintenance (59). In addition, TCF3, one of the key Wnt-activated transcriptional regulators, is proposed to regulate the expression of key ESC transcription factors including Oct4 and Nanog, and co-occupies almost all promoter regions occupied by ESC-specific transcription factors $(58,60)$. $\beta$-Catenin is reported to form a complex with Oct4 in a TCF-independent manner to enhance ESC pluripotency (61). However, $\beta$-catenin is also suggested to be unimportant for pluripotent stem cell self-renewal and expansion (62-64), indicating that the effect of $\beta$-catenin may be context-dependent.

The secretin receptor (SR) is a family of GPCRs that interact with secretin, the gastrointestinal peptide hormone $(11,65)$. Secretin is a member of the secretin-glucagon family and regulates the secretion of bicarbonate ions into the duodenum from the epithelia lining the pancreatic and biliary ducts. In addition to regulating water homeostasis, secretin is reported to have pleiotropic effects in several organ systems and has been termed a neuroendocrine hormone. Vasoactive intestinal peptide (VIP) and the pituitary adenylyl cyclase activating polypeptides (PACAPs) belong to the secretin peptide family and display pleiotropic functions (66). The physiological actions of these widely distributed peptides are reported to be through the stimulation of three common GPCRs: Vasoactive intestinal polypeptide receptor 1 (VPAC1), VPAC2 and phosphatase of activated cells 1 receptor (PAC1R). VPAC1, VPAC2 and PAC1R preferentially activate adenylyl cyclase and increase intracellular cAMP, thereby regulating stem cell maintenance.

\section{GPCRs IN SOMATIC REPROGRAMMING TO PSCs}

The iPSC field advanced significantly in 2006 when Takahashi and Yamanaka (2) discovered that retroviral transduction of only four genes-Sox2, Oct4, c-Myc, and Klf4-was sufficient to induce pluripotency in somatic cells through somatic reprogramming to PSCs. Importantly, iPSCs provide an alternative to the use of human embryos, overcoming ethical issues. In addition, iPSC technology allows the use of patientspecific somatic cells to generate therapeutic iPSCs, overcoming the potential for immune rejection. In human cells, re- 
programming using the additional factors Nanog and Lin28 to replace c-Myc and Klf4 dramatically reduces the time required to generate iPSCs (3). To avoid genetic modification and to improve the efficiency of iPSC generation and differentiation, iPSC production technology was advanced using techniques that avoided stable integration of foreign genetic material into the host genome $(46,67)$. Treatment of synthetic compounds (small molecules) such as valproic acid favors the maintenance and induction of the pluripotent state, enhancing somatic reprogramming to PCSs $(2,3,46)$. The goal of patient-specific iPSC therapy is to prepare somatic cells from patients, reprogram and differentiate them to replace diseased cells, and successfully transplant them back into the same patients without immune problems. Because pluripotent colony morphology is closely associated with the maintenance of pluripotency, the mechanisms through which these colonies form and organize may be important for managing somatic cell reprogramming (68). Our results showed that 81 GPCRs are differentially expressed during somatic reprogramming to iPSCs (Fig. 1B and Table 1). Of these GPCRs, several have confirmed roles in regulating the self-renewal and other properties of PCSs. Several GPCRs have been proposed to have crucial roles in somatic reprogramming to PCSs.

Somatic reprogramming to iPSCs requires dramatic morphological and organizational cell changes (69). Interestingly, the inhibition of Gi-coupled receptor signaling with pertussis toxin retracts stem cell colonies inward into a dense multilayered conformation without affecting proliferation, survival, or pluripotency (70). Activation of Gs-coupled receptor signaling with cholera toxin did not affect colony morphology, suggesting that Gi-coupled GPCRs may play a role in some aspects of somatic cell reprogramming to PSCs.

In mouse ESCs, mGluR5 activation promotes self-renewal through interactions with the LIF signaling pathway $(24,71)$. Various mGluR subtypes are involved in differentiation. Specifically, the differentiation of mouse ESCs into embryoid bodies is associated with the induction of mGluR4, which promotes differentiation of GABAergic neurons $(25,72)$. Other GPCRs are found in ESCs, but very few studies have described their functional roles.

Specific lipids regulate various features of ESCs by binding to GPCRs such as rhodopsin family LPA and S1P receptors (40). Both S1P and LPA receptors are positive modulators of ESC maintenance through ERK- and $\mathrm{Ca}^{2+}$-mediated pathways $(40,73)$. ESCs express LPA and S1P receptor subtypes $(42,73)$. The LPA and S1P signaling pathways that affect ESC maintenance and somatic reprogramming to PSCs activate the ERK signaling pathway via GPCR/Gi-, protein kinase $\mathrm{C}_{-}$, and c-Src-dependent mechanisms (40).

The adhesion GPCR, GPR125, is a potential marker for somatic reprogramming to PCSs. GPR125 is an orphan adhesion-type GPCR also known as a germ-line progenitor marker (74) and tumor endothelial marker 5-like. GPR125 is found in proliferative adult spermatogonial progenitor cells and multi- potent adult spermatogonial-derived stem cells but is down-regulated after differentiation (74). Members of the tumor endothelial marker family have been identified by locating genes with elevated expression during tumor angiogenesis (75). A recent study revealed that GPCRs display dramatic differences in expression when cells differentiate (21). The authors generated a real-time polymerase chain reaction-based expression profile of 343 GPCRs in ESCs and revealed that 161 of them were expressed at low levels in undifferentiated ESCs, 30 were moderately expressed, and 7 were highly expressed. GPR125 was one of the important GPCRs expressed differentially in ESCs.

The Wnt/FZD signaling pathway is reported to promote reprogramming of somatic cells to PSCs $(55,76)$. Addition of soluble Wnt3a or expression of constitutively active $\beta$-catenin, a downstream component of the Wnt pathway, promotes reprogramming of fibroblasts to pluripotency (76). Recent study revealed that Wnt3a and GSK-3 $\beta$ inhibitors successfully stimulate reprogramming whereas $\mathrm{Wnt} / \beta$-catenin enhances iPSCs induction at the early stage of reprogramming by interacting with reprogramming factors Klf4, Oct4, and Sox2, further increasing the expression of pluripotency circuitry genes (77). Without activation of reprogramming factors, Wnt3a/ $\beta$-catenin activation cannot return differentiated cells to iPSCs. However, with the help of ectopic reprogramming factors, $\beta$-catenin can promote the activation of the pluripotent network pathway at the early reprogramming stage, leading to enhanced iPS cell generation. This indicates that $\beta$-catenin is required for somatic reprogramming to pluripotency but not required for pluripotency maintenance.

\section{GPCR SIGNALING IN SOMATIC REPROGRAMMING TO CSCs}

CSCs were first discovered in 1994 when a cell type with low abundance derived from human acute myeloid leukemia successfully caused cancer in mice (78). The origin of CSCs is still being debated; particularly whether they represent a stromal stem cell that has undergone some sort of malignant change or whether they are differentiated cells that acquire stemness as part of the malignant somatic reprogramming or transformation process. According to the CSC hypothesis, only a small fraction of immature cellular intermediates is responsible for mediating tumor expansion, resistance, and metastases. Thus, CSCs are the tumor component that diffuses out of the organ, where it cannot be eliminated by surgical therapy and causes relapses resistant to chemotherapy and radiotherapy (79). The presence of CSCs has important implications for treatment, as current therapies may target the bulk of tumor cells but overlook CSCs, resulting in tumor recurrence. Consequently, future treatments specifically targeting CSCs may be more effective.

Tumor-suppressor genes are often lacking in many CSCs, and this absence may explain the capacity CSCs for self-renew- 
al (79). FZD and chemokine receptors are among the receptors that collect signals within the environmental niche. Our results showed that 195 GPCRs are differentially expressed during transformation to CSCs or during CSC sphere formation (Fig. 1C, 1D, and Table 1). Of these GPCRs, several have confirmed roles in regulating the self-renewal and other properties of CSCs. Several GPCRs may have crucial roles in the transformation to CSCs.

Cancer cells or CSCs generate a supportive microenvironment by activating various proliferation and stress signaling pathways of normal cells (16). Conversely, stromal cells, such as cancer-associated fibroblasts or tumor-associated macrophages, promote tumor progression by secreting growth factors, chemokines, and pro-migratory extracellular matrix components (8). In particular, tumor-surrounding adipocytes exhibit a specific and activated phenotype. These cells have been designated cancer-associated adipocytes, and they contribute to the progression of various tumors by stimulating local and distant metastasis (80). Several fatty acid-sensing rhodopsin family GPCRs, such as GPR35, GPR41 (free fatty acid receptor 3, FFAR3), GPR43 (free fatty acid receptor 2, FFAR2), GPR81 (hydroxycarboxylic acid receptor 1, HCAR1), and GPR120, are usually expressed in tumor microenvironments and are suggested to be involved in somatic reprogramming to CSCs.

The rhodopsin family chemokine receptors are centrally linked to the organ-specific metastasis of a number of cancers, in line with their normal immune cell function of directing receptor-bearing leukocytes toward the sites of chemokine production. Similarly, tumor cells abnormally expressing chemokine receptors can induce the migratory activity of chemokines, facilitating metastasis to other organs (8). Recent study revealed that although both CXCR4 and CXCR7 have roles in stem cell therapy for remyelination, the two SDF-1-interacting chemokine receptors also play different and opposing roles in tumor metastasis, with CXCR4 mediating breast cancer invasion and CXCR7 impairing invasion but enhancing primary tumor growth through angiogenesis $(32,81,82)$. CXCR4 is included among stem cell markers (83), and evidence of its importance in tumor development is rapidly emerging (84). CXCR4 is expressed in various human cancers, including breast cancer, and carcinoma-associated fibroblasts are a key source of SDF-1 in the tumor microenvironment. Recent studies have revealed that high levels of SDF-1 expression is closely correlated with poor prognosis of breast cancer patients and CXCR4 signaling has contrasting effects on normal and malignant breast stem cell activity, demonstrating that it specifically regulates breast CSC activities (85). CXCR4 activates Rac1 through P-REX1, which plays a central role in metastasis in most types of breast cancer (40). CXCR4 can also couple to G12/13 in basal-like breast cancer cells, in which Ga13 protein expression is highly up-regulated, thus driving metastasis through a Ga12/13-RhoA-dependent mechanism (86) similar to that of LPA and PAR-1 receptors, all of which can be considered potential targets for metastasis prevention and treatment. Like CXCR4, CXCR7 is also overexpressed in human breast cancer and implicated in enhancing cancer cell adhesion to fibronectin and endothelial cells by activating PI3K and MAPK (82). CXCR4 and CXCR7 can form both homodimers and heterodimers and the heterodimer formation may modulate CXCR4 signalling both positively and negatively (87). Other chemokine receptors, including CCR7 and CCR10, have also been shown to have direct roles in the metastatic homing of cancer cells as well as cancer cell survival and growth (8). Chemokines may enhance cytokine-rich microenvironments and induce the release of matrix metalloproteases, which facilitate tumor cell survival, proliferation, and invasion.

Expression of the rhodopsin family CB receptors is upregulated in many cancers including breast and prostate cancers. Activation of the receptors led to decreased cell viability, increased apoptosis, and decreased androgen or estrogen receptor expression (88). Clinical studies utilizing cannabinoids for patients with metastatic cancers may take advantage not only of its beneficial effects on cancer but also of their analgesic properties for metastatic cancer pain. CB receptors also modulate the differentiation of glioma stem-like cells, supporting a possible anti-cancer role for cannabinoid receptors (89).

In human mammals, there are two types of rhodopsin family melatonin receptors: MT1 (Mel1A) and MT2 (Mel1B). Melatonin is reported to suppress breast cancer cell proliferation by inhibiting the up-regulation of estrogen-induced cyclin D1 via MT1 and down-regulating estrogen receptor alpha (90). 6-Hydroxymelatonin, an oxidated form of melatonin, has also been shown to bind selectively to MT1 and to have antioxidant properties (91).

The purinergic system plays a key role in cell growth in glioblastoma, the most common and aggressive tumor of the brain, and may be characterized by a CSC subpopulation (92). Some purinergic receptors, such as $\mathrm{P} 1$ and $\mathrm{P} 2 \mathrm{Y}$, are rhodopsin family GPCRs and their expressions have been differently expressed in tumor spheres containing markers for CSCs, suggesting that the purinergic system affects CSC biology.

The Wnt and FZD pathways are involved in various differentiation events during embryonic development and tumor formation (93). During malignant progression, cancers actively rearrange the extracellular matrix and tumor stroma to create suitable microenvironments. In cancers, the Wnt and FZD pathways are typically associated with a transition from epithelial to mesenchymal cellular stages as the tumor develops during metastasis (94). Members of the FZD family activate upstream signaling that results in epithelial-mesenchymal transitions (79).

In addition, recent studies have uncovered a role for GPR116, a member of the poorly characterized family of adhesion GPCRs, in the invasion and migration of breast cancer cells via a Goq-RhoA/Rac1-dependent mechanism (95). 


\section{SUMMARY}

The molecular functions of GPCR signaling pathways have been thoroughly studied for more than a century from the perspectives of both basic science and therapeutic applications. In this review, we addressed the current understanding of the role of GPCRs in stem cell maintenance and somatic reprogramming to PCSs or CSCs. Differential GPCRs play crucial roles not only in tumor progression, adipogenesis, and inflammation, but also in embryonic development and stem cell maintenance. Recent studies demonstrate the important roles of various GPCRs in the regulation of self-renewal and other biological properties of PCSs or CSCs during maintenance. Differential expression profiles of GPCRs may also be detected during somatic reprogramming to iPSCs or CSCs and during CSC sphere formation, indicating that specific GPCRs have key roles in somatic reprogramming to iPSCs and transformation to CSCs via regulation of cell survival, proliferation, migration, and self-renewal of PCSs and CSCs. A more universal definition of the general systems through which GPCRs exert their numerous physiological and pathological roles is necessary to appreciate the overall implications for somatic reprogramming and tumorigenesis. In particular, extensive cross talk and co-regulation may occur between GPCR- and tyrosine kinase growth factor receptor-initiated signaling pathways and through receptor transactivation. Given the evidence discussed herein, the significance of the roles of differential GPCRs in stem cell maintenance and somatic reprogramming to PCSs or CSCs will be manifested in various biological and developmental phenomena. Modulation of various GPCRs' expression and subcellular localizations may have important implications for human health. A systems-level understanding may provide the necessary molecular framework for the development of novel approaches to therapeutic intervention in some of the most prevalent human diseases.

\section{ACKNOWLEDGEMENTS}

This work was supported by grants from the National Research Foundation (NRF) funded by the Korean Ministry of Education, Science and Technology (MEST) (no. 2010-0020348 and no. 2013M3A9D3045880).

\section{REFERENCES}

1. Russ AP, Wattler S, Colledge WH et al (2000) Eomesodermin is required for mouse trophoblast development and mesoderm formation. Nature 404, 95-99

2. Takahashi K and Yamanaka S (2006) Induction of pluripotent stem cells from mouse embryonic and adult fibroblast cultures by defined factors. Cell 126, 663-676

3. Yu J, Vodyanik MA, Smuga-Otto K et al (2007) Induced pluripotent stem cell lines derived from human somatic cells. Science 318, 1917-1920

4. Clarke MF and Fuller M (2006) Stem cells and cancer: two faces of eve. Cell 124, 1111-1115

5. Dirks PB (2006) Cancer: stem cells and brain tumours. Nature 444, 687-688

6. Pierce KL, Premont RT and Lefkowitz RJ (2002) Seventransmembrane receptors. Nat Rev Mol Cell Biol 3, 639-650

7. Dorsam RT and Gutkind JS (2007) G-protein-coupled receptors and cancer. Nat Rev Cancer 7, 79-94

8. O'Hayre M, Degese MS and Gutkind JS (2014) Novel insights into $G$ protein and $G$ protein-coupled receptor signaling in cancer. Curr Opin Cell Biol 27, 126-135

9. Overington JP, Al-Lazikani B and Hopkins AL (2006) How many drug targets are there? Nat Rev Drug Discov 5, 993-996

10. Takeda S, Kadowaki S, Haga T, Takaesu H and Mitaku S (2002) Identification of $G$ protein-coupled receptor genes from the human genome sequence. FEBS Lett 520, 97-101

11. Fredriksson R, Lagerstrom MC, Lundin LG and Schioth HB (2003) The G-protein-coupled receptors in the human genome form five main families. Phylogenetic analysis, paralogon groups, and fingerprints. Mol Pharmacol 63, 1256-1272

12. Gloriam DE, Fredriksson R and Schioth HB (2007) The G protein-coupled receptor subset of the rat genome. BMC Genomics 8, 338

13. Taussig R, Iniguez-Lluhi JA and Gilman AG (1993) Inhibition of adenylyl cyclase by Gi alpha. Science 261, 218221

14. Hubbard KB and Hepler JR (2006) Cell signalling diversity of the Gqalpha family of heterotrimeric $G$ proteins. Cell Signal 18, 135-150

15. Sassone-Corsi P (2012) The cyclic AMP pathway. Cold Spring Harb Perspect Biol 4, 1-3

16. Gutkind JS (1998) The pathways connecting G protein-coupled receptors to the nucleus through divergent mitogen-activated protein kinase cascades. J Biol Chem 273, 1839-1842

17. Han D, Kim HJ, Choi HY et al (2014) 3,2'-Dihydroxyflavone-treated Pluripotent Stem Cells Show Enhanced Proliferation, Pluripotency Markers Expression, and Neuroprotective Properties. Cell Transplant [Epub ahead of print].

18. Jeon K, Oh HJ, Lim H et al (2012) Self-renewal of embryonic stem cells through culture on nanopattern polydimethylsiloxane substrate. Biomaterials 33, 5206-5220

19. Jeon K, Lim H, Kim JH et al (2012) Bax inhibitor-1 enhances survival and neuronal differentiation of embryonic stem cells via differential regulation of mitogen-activated protein kinases activities. Biochim Biophys Acta 1823, 2190-2200

20. Nakamura K, Salomonis N, Tomoda K, Yamanaka S and Conklin BR (2009) G(i)-coupled GPCR signaling controls the formation and organization of human pluripotent colonies. PLoS One 4, e7780

21. Layden BT, Newman M, Chen F, Fisher A and Lowe WL Jr (2010) G protein coupled receptors in embryonic stem cells: a role for Gs-alpha signaling. PLoS One 5, e9105

22. Callihan P, Mumaw J, Machacek DW, Stice SL and Hooks SB (2011) Regulation of stem cell pluripotency and differentiation by $\mathrm{G}$ protein coupled receptors. Pharmacol Ther 129, 290-306 
23. Melchiorri D, Cappuccio I, Ciceroni C et al (2007) Metabotropic glutamate receptors in stem/progenitor cells. Neuropharmacology 53, 473-480

24. Cappuccio I, Spinsanti P, Porcellini A et al (2005) Endogenous activation of mGlu5 metabotropic glutamate receptors supports self-renewal of cultured mouse embryonic stem cells. Neuropharmacology 49 Suppl 1, 196-205

25. Cappuccio I, Verani R, Spinsanti P et al (2006) Contextdependent regulation of embryonic stem cell differentiation by mGlu4 metabotropic glutamate receptors. Neuropharmacology 51, 606-611

26. Ciceroni C, Mosillo P, Mastrantoni E et al (2010) mGLU3 metabotropic glutamate receptors modulate the differentiation of SVZ-derived neural stem cells towards the astrocytic lineage. Glia 58, 813-822

27. Schwirtlich M, Emri Z, Antal K, Mate Z, Katarova Z and Szabo G (2010) GABA(A) and GABA(B) receptors of distinct properties affect oppositely the proliferation of mouse embryonic stem cells through synergistic elevation of intracellular $\mathrm{Ca}(2+)$. FASEB J 24, 1218-1228

28. Teng L, Tang YB, Sun F et al (2013) Non-neuronal release of gamma-aminobutyric Acid by embryonic pluripotent stem cells. Stem Cells Dev 22, 2944-2953

29. Bachelerie F, Ben-Baruch A, Burkhardt AM et al (2014) International Union of Basic and Clinical Pharmacology. [corrected]. LXXXIX. Update on the extended family of chemokine receptors and introducing a new nomenclature for atypical chemokine receptors. Pharmacol Rev 66, $1-79$

30. Southgate TD, McGinn OJ, Castro FV et al (2010) CXCR4 mediated chemotaxis is regulated by 5T4 oncofetal glycoprotein in mouse embryonic cells. PLoS One 5, e9982

31. Carbajal KS, Schaumburg C, Strieter R, Kane J and Lane TE (2010) Migration of engrafted neural stem cells is mediated by CXCL12 signaling through CXCR4 in a viral model of multiple sclerosis. Proc Natl Acad Sci U S A 107, 11068-11073

32. Williams JL, Patel JR, Daniels BP and Klein RS (2014) Targeting CXCR7/ACKR3 as a therapeutic strategy to promote remyelination in the adult central nervous system. J Exp Med 211, 791-799

33. Piomelli D (2003) The molecular logic of endocannabinoid signalling. Nat Rev Neurosci 4, 873-884

34. Brown AJ (2007) Novel cannabinoid receptors. Br J Pharmacol 152, 567-575

35. Jiang S, Fu Y, Williams J et al (2007) Expression and function of cannabinoid receptors CB1 and CB2 and their cognate cannabinoid ligands in murine embryonic stem cells. PLoS One 2, e641

36. Rossi F, Bernardo ME, Bellini G et al (2013) The cannabinoid receptor type 2 as mediator of mesenchymal stromal cell immunosuppressive properties. PLoS One 8, e80022

37. Galve-Roperh I, Chiurchiu V, Diaz-Alonso J, Bari M, Guzman M and Maccarrone M (2013) Cannabinoid receptor signaling in progenitor/stem cell proliferation and differentiation. Prog Lipid Res 52, 633-650

38. Gowran A, McKayed K and Campbell VA (2013) The cannabinoid receptor type 1 is essential for mesenchymal stem cell survival and differentiation: implications for bone health. Stem Cells Int 2013, 796715
39. Mclntyre TM, Pontsler AV, Silva AR et al (2003) Identification of an intracellular receptor for lysophosphatidic acid (LPA): LPA is a transcellular PPARgamma agonist. Proc Natl Acad Sci U S A 100, 131-136

40. Rodgers A, Mormeneo D, Long JS, Delgado A, Pyne NJ and Pyne S (2009) Sphingosine 1-phosphate regulation of extracellular signal-regulated kinase-1/2 in embryonic stem cells. Stem Cells Dev 18, 1319-1330

41. Schuck S, Soloaga A, Schratt G, Arthur JS and Nordheim A (2003) The kinase MSK1 is required for induction of c-fos by lysophosphatidic acid in mouse embryonic stem cells. BMC Mol Biol 4, 6

42. Pebay A, Wong RC, Pitson SM et al (2005) Essential roles of sphingosine-1-phosphate and platelet-derived growth factor in the maintenance of human embryonic stem cells. Stem Cells 23, 1541-1548

43. Whetton AD, Lu Y, Pierce A, Carney L and Spooncer E (2003) Lysophospholipids synergistically promote primitive hematopoietic cell chemotaxis via a mechanism involving Vav 1. Blood 102, 2798-2802

44. Jaganathan BG, Ruester B, Dressel L et al (2007) Rho inhibition induces migration of mesenchymal stromal cells. Stem Cells 25, 1966-1974

45. Donati C, Cencetti F, Nincheri P et al (2007) Sphingosine 1-phosphate mediates proliferation and survival of mesoangioblasts. Stem Cells 25, 1713-1719

46. Song M, Paul S, Lim H, Dayem AA and Cho SG (2012) Induced pluripotent stem cell research: a revolutionary approach to face the challenges in drug screening. Arch Pharm Res 35, 245-260

47. Xu XX, Zhang LH and Xie X (2014) Somatostatin receptor type 2 contributes to the self-renewal of murine embryonic stem cells. Acta Pharmacol Sin 35, 1023-1030

48. Schioth HB, Nordstrom KJ and Fredriksson R (2010) The adhesion GPCRs; gene repertoire, phylogeny and evolution. Adv Exp Med Biol 706, 1-13

49. Yona S, Lin HH, Siu WO, Gordon S and Stacey M (2008) Adhesion-GPCRs: emerging roles for novel receptors. Trends Biochem Sci 33, 491-500

50. Bai Y, Du L, Shen L, Zhang Y and Zhang L (2009) GPR56 is highly expressed in neural stem cells but downregulated during differentiation. Neuroreport 20, 918-922

51. Formstone CJ and Little PF (2001) The flamingo-related mouse Celsr family (Celsr1-3) genes exhibit distinct patterns of expression during embryonic development. Mech Dev 109, 91-94

52. Seandel M, Falciatori I, Shmelkov SV, Kim J, James D and Rafii S (2008) Niche players: spermatogonial progenitors marked by GPR125. Cell Cycle 7, 135-140

53. Foord SM, Bonner $\mathrm{TI}$, Neubig RR et al (2005) International Union of Pharmacology. XLVI. G protein-coupled receptor list. Pharmacol Rev 57, 279-288

54. Wang HY, Liu T and Malbon CC (2006) Structure-function analysis of Frizzleds. Cell Signal 18, 934-941

55. Nusse R (2008) Wnt signaling and stem cell control. Cell Res 18, 523-527

56. Cai L, Ye Z, Zhou BY, Mali P, Zhou C and Cheng L (2007) Promoting human embryonic stem cell renewal or differentiation by modulating Wnt signal and culture conditions. Cell Res 17, 62-72 
57. Walsh J and Andrews PW (2003) Expression of Wnt and Notch pathway genes in a pluripotent human embryonal carcinoma cell line and embryonic stem cell. APMIS 111, 197-210; discussion 210-191

58. Ding VM, Ling L, Natarajan S, Yap MG, Cool SM and Choo AB (2010) FGF-2 modulates Wnt signaling in undifferentiated hESC and iPS cells through activated PI3-K/GSK3beta signaling. J Cell Physiol 225, 417-428

59. Melchior K, Weiss J, Zaehres $\mathrm{H}$ et al (2008) The WNT receptor FZD7 contributes to self-renewal signaling of human embryonic stem cells. Biol Chem 389, 897-903

60. Tam WL, Lim CY, Han J et al (2008) T-cell factor 3 regulates embryonic stem cell pluripotency and self-renewal by the transcriptional control of multiple lineage pathways. Stem Cells 26, 2019-2031

61. Kelly KF, Ng DY, Jayakumaran G, Wood GA, Koide H and Doble BW (2011) beta-catenin enhances Oct-4 activity and reinforces pluripotency through a TCF-independent mechanism. Cell Stem Cell 8, 214-227

62. Lyashenko $N$, Winter M, Migliorini D, Biechele T, Moon RT and Hartmann C (2011) Differential requirement for the dual functions of beta-catenin in embryonic stem cell self-renewal and germ layer formation. Nat Cell Biol 13, 753-761

63. Yi F, Pereira L, Hoffman JA et al (2011) Opposing effects of Tcf3 and Tcf1 control Wnt stimulation of embryonic stem cell self-renewal. Nat Cell Biol 13, 762-770

64. Miki T, Yasuda SY and Kahn M (2011) Wnt/beta-catenin signaling in embryonic stem cell self-renewal and somatic cell reprogramming. Stem Cell Rev 7, 836-846

65. Afroze S, Meng F, Jensen K et al (2013) The physiological roles of secretin and its receptor. Ann Transl Med 1, 29

66. Dickson L and Finlayson K (2009) VPAC and PAC receptors: From ligands to function. Pharmacol Ther 121, 294-316

67. Lee $\mathrm{CH}$, Kim JH, Lee HJ et al (2011) The generation of iPS cells using non-viral magnetic nanoparticle based transfection. Biomaterials 32, 6683-6691

68. Yang W, Wei W, Shi C et al (2009) Pluripotin combined with leukemia inhibitory factor greatly promotes the derivation of embryonic stem cell lines from refractory strains. Stem Cells 27, 383-389

69. Maherali N and Hochedlinger K (2008) Guidelines and techniques for the generation of induced pluripotent stem cells. Cell Stem Cell 3, 595-605

70. Teng HF, Kuo YL, Loo MR et al (2010) Valproic acid enhances Oct4 promoter activity in myogenic cells. J Cell Biochem 110, 995-1004

71. Spinsanti P, De Vita T, Di Castro S et al (2006) Endogenously activated mGlu5 metabotropic glutamate receptors sustain the increase in c-Myc expression induced by leukaemia inhibitory factor in cultured mouse embryonic stem cells. J Neurochem 99, 299-307

72. Sarichelou I, Cappuccio I, Ferranti F et al (2008) Metabotropic glutamate receptors regulate differentiation of embryonic stem cells into GABAergic neurons. Cell Death Differ 15, 700-707

73. Todorova MG, Fuentes E, Soria B, Nadal A and Quesada I (2009) Lysophosphatidic acid induces $\mathrm{Ca} 2+$ mobilization and c-Myc expression in mouse embryonic stem cells via the phospholipase C pathway. Cell Signal 21, 523-528

74. Seandel M, James D, Shmelkov SV et al (2007) Generation of functional multipotent adult stem cells from GPR125 + germline progenitors. Nature 449, 346-350

75. Carson-Walter EB, Watkins DN, Nanda A, Vogelstein B, Kinzler KW and St Croix B (2001) Cell surface tumor endothelial markers are conserved in mice and humans. Cancer Res 61, 6649-6655

76. Marson A, Foreman R, Chevalier B et al (2008) Wnt signaling promotes reprogramming of somatic cells to pluripotency. Cell Stem Cell 3, 132-135

77. Zhang P, Chang WH, Fong B et al (2014) Regulation of induced pluripotent stem (iPS) cell induction by Wnt/beta-catenin signaling. J Biol Chem 289, 9221-9232

78. Bonnet D and Dick JE (1997) Human acute myeloid leukemia is organized as a hierarchy that originates from a primitive hematopoietic cell. Nat Med 3, 730-737

79. Rodriguez-Pinilla SM, Sarrio D, Moreno-Bueno G et al (2007) Sox2: a possible driver of the basal-like phenotype in sporadic breast cancer. Mod Pathol 20, 474-481

80. Prevarskaya N, Skryma R and Shuba Y (2011) Calcium in tumour metastasis: new roles for known actors. Nat Rev Cancer 11, 609-618

81. Dean $M$ (2006) Cancer stem cells: redefining the paradigm of cancer treatment strategies. Mol Interv 6, 140-148

82. Hernandez L, Magalhaes MA, Coniglio SJ, Condeelis JS and Segall JE (2011) Opposing roles of CXCR4 and CXCR7 in breast cancer metastasis. Breast Cancer Res 13, R128

83. Wu X, Lee VC, Chevalier E and Hwang ST (2009) Chemokine receptors as targets for cancer therapy. Curr Pharm Des 15, 742-757

84. Lazennec G and Richmond A (2010) Chemokines and chemokine receptors: new insights into cancer-related inflammation. Trends Mol Med 16, 133-144

85. Ablett MP, O'Brien CS, Sims AH, Farnie G and Clarke RB (2014) A differential role for CXCR4 in the regulation of normal versus malignant breast stem cell activity. Oncotarget 5, 599-612

86. Burdon T, Smith A and Savatier P (2002) Signalling, cell cycle and pluripotency in embryonic stem cells. Trends Cell Biol 12, 432-438

87. Levoye A, Balabanian K, Baleux F, Bachelerie F and Lagane B (2009) CXCR7 heterodimerizes with CXCR4 and regulates CXCL12-mediated G protein signaling. Blood $113,6085-6093$

88. Sarfaraz S, Adhami VM, Syed DN, Afaq F and Mukhtar H (2008) Cannabinoids for cancer treatment: progress and promise. Cancer Res 68, 339-342

89. Aguado T, Carracedo A, Julien B et al (2007) Cannabinoids induce glioma stem-like cell differentiation and inhibit gliomagenesis. J Biol Chem 282, 6854-6862

90. Rogelsperger O, Ekmekcioglu C, Jager W et al (2009) Coexpression of the melatonin receptor 1 and nestin in human breast cancer specimens. J Pineal Res 46, 422-432

91. Tan DX, Manchester LC, Terron MP, Flores LJ and Reiter RJ (2007) One molecule, many derivatives: a never-ending interaction of melatonin with reactive oxygen and nitrogen species? J Pineal Res 42, 28-42

92. Ledur PF, Villodre ES, Paulus R, Cruz LA, Flores DG and Lenz G (2012) Extracellular ATP reduces tumor sphere 
G protein-coupled receptors in stem cell maintenance and somatic reprogramming to pluripotent or cancer stem cells Hye Yeon Choi, et al.

growth and cancer stem cell population in glioblastoma cells. Purinergic Signal 8, 39-48

93. Lonardo E, Hermann PC and Heeschen C (2010) Pancreatic cancer stem cells - update and future perspectives. Mol Oncol 4, 431-442

94. MacDonald BT, Tamai K and He X (2009) Wnt/beta-cat- enin signaling: components, mechanisms, and diseases. Dev Cell 17, 9-26

95. Moriconi A, Cesta MC, Cervellera MN et al (2007) Design of noncompetitive interleukin-8 inhibitors acting on CXCR1 and CXCR2. J Med Chem 50, 3984-4002 